\title{
El poder de la presión: la prórroga permanente e incondicionada del Tratado de No Proliferación de Armas Nucleares
}

\author{
Julio C. Carasales*
}

En abril-mayo de 1995 tuvo lugar en Nueva York una importante conferencia que debió decidir si el Tratado de No Proliferación ởe Armas Nucleares debía ser renovado indefinidamente o por períodos sucesivos. Se impuso la primera de esas posiciones, pero ello se debió, en buena medida, a una presión intensa y continuada ejercida por el principal interesado, o sea Estados Unidos. Si bien el uso de presión es un fenómeno normal en las reuniones internacionales, todo indica que en la conferencia del TNP la misma alcanzó un nivel desproporcionado.

Entre los días 17 de abril y 12 de mayo de 1995 tuvo lugar en Nueva York una de las conferencias internacionales más importantes de los últimos años, que debía decidir si el Tratado de No Proliferación de Armas Nucleares perduraria indefinidamente y sin condiciones, o si su validez sería renovada periódicamente, cada tantos años, según el progreso que se alcanzara en otras materias conexas, en especial el desarme nuclear.

\footnotetext{
- Este artículo refleja puntos de vista personales del autor y no de la institución a que pertenece. Asimismo, cabe advertir que las palabras "paises nucleares" y "paises no nucleares" se utilizan, en aras de la brevedad, en lugar de las expresiones correctas "paises dueños de armas nucleares" y "países no dueños de armas nucleares".
} 
Como es sabido, esa conferencia resolvió finalmente que el Tratado de No Proliferación (TNP) continuara en vigencia sine die e incondicionalmente. El duro y dificil enfrentamiento que se esperaba entre dos grupos de países, uno que quería lo que en definitiva se adoptó, y otro -que se presumía más numeroso-que propugnaba la extensión periódica del TNP, sujeta explícita o implícitamente a determinados requisitos, distó de tener las características auguradas. La pugna se dio, pero entre dos fuerzas disparejas, revelándosé sin duda como la más débil justamente la que todo indicaba sería la mayoritaria y la que mejor reflejaba la opinión pública mundial. Incluso llamó la atención la rapidez con que los partidarios de esta última posición fueron quedando cada vez más aislados.

Esa situación fue inesperada, ${ }^{1}$ pero como nada en política carece de causa, aunque frecuentemente no sea fácil detectarla, cabe preguntarse cuál fue la razón de ese sorpresivo desarrollo de la denominada Conferencia de Extensión del TNP. La respuesta puede ser variada pero, básicamente, en el origen se halla la presión diplomática de larga data, creciente, vigorosa y sostenida, que Estados Unidos ejerció para conseguir el resultado que convenía a sus intereses nacionales.

El presente artículo pretende fundamentar la precedente afirmación. Previamente, debe hacerse dos salvedades: por una parte, hay que reconocer que la presión se ejerce normalmente en ámbitos reservados, o por lo menos no públicos, y mucho menos por escrito, por lo que rara vez es posible documentarla; por la otra, que la presión diplomática es un arma común en el manejo de las relaciones internacionales, por lo que su ejercicio no tiene en sí mismo una connotación peyorativa. El autor no abriga un propósito crítico; simplemente desea poner de manifiesto su empleo en este caso, que prueba una vez más que cuando la presión es efectuada por un Estado de extremo poderio, que le otorga alto relieve y utiliza la gama de recursos "convincentes" a su disposición, esa presión es prácticamente irresistible.

\footnotetext{
${ }^{1}$ La decisión de extender indefinidamente el TNP "confundió a los observadores más pesimistas; incluso muchos de los más optimistas proponentes de la extensión indefinida quedaron sorprendidos por la magnitud final del apoyo a esa decisión y por el hecho de que fue adoptada sin un voto potencialmente divisivo". Asi describe lo ocurrido un conocido especialista, Lewis Dunn, en su articulo "High noon for the NPT", publicado en Arms control today, Volume 25, Number 6 , Washington D.C., July-August 1995, p. 3
} 


\section{Antecedentes.}

¿Por qué el Tratado de No Prouliferación debió ser objeto de una conferencia que decidiera su renovación, cuando la regla es que las convenciones sobre desarme tengan una duración indefinida?

Para encontrar la razón tenemos que remontarnos más de treinta años atrás, cuando se negoció el TNip

El problema se inicia en 1945, con el estallido de la primera bomba nuclear en Hiroshima. Seguidamente, en pocos años se producen dos fenómenos inquietantes: nuevos desarrollos del arma nuclear multiplican tanto su capacidad destructiva que los artefactos utilizados en Hiroshima y Nagasaki parecen juegos de niños; $y$, paralelamente, cuatro nuevos países se unen a Estados Unídos en la posesión de esos instrumentos de destrucción en masa: la Unión Soviética en 1.949, Gran Bretaña en 1952, Francia en 1960 y China en 1964.

La situación se hizo cada vez más preocupante. La conocida frase del Presidente Kennedy en 1963 la reflejó adecuadamente, cuando dijo: "Estoy obsesionado por la sensación de que en 1970 ... puede haber diez potencias nucleares en vez de cinco, y hacia 1975, 15 ó 20 ". Es indudable que un mundo en que esas terroríficas armas existieran en los arsenales de una cantidad crecida y creciente de países seria cada vez más peligroso e inseguro, por lo que su proliferación fue, justificamente, materia de la inquietud de toda la comunidad internacional, aunque por cierto algunos de sus miembros desearan poder esgrimir también ese instrumento bélico que daba prestigio y poder.

Al mismo tiempo, quienes lo tenían, y en primer lugar las dos superpotencias de entonces, Estados Unidos y la Unión Soviética, tampoco veían con buenos ojos la posibilidad de que surgieran nuevos competidores que desafiaran su poderio, un poderío basado, en parte, en la posesión de armas nucleares.

Resultó natural entonces que se tratara de preparar un tratado internacional que prohibiera que las armas nucleares "proliferaran", es decir, que aparecieran en manos de otros Estados además de los cinco que ya las tenían. La negociación de ese tratado se efectuó hacia mediados de la década de 1960 en Ginebra, en el seno del Comité de Desarme de las Dieciocho Naciones. ${ }^{2}$ Quizá el término "negociación" no sea realmente el apropiado, porque si bien hubo años de conversaciones, la verdadera

\footnotetext{
${ }^{2}$ En realidad, fueron diecisiete, ya que Francia nunca ocupó el puesto que le estaba asignado.
} 
"negociación" tuvo lugar entre Estados Unidos y la Unión Soviética, que en plena Guerra Fría discordaban en todo salvo en impedir que el arma nuclear pasara a poder también de otros Estados, en primer lugar Alemania y Japón. Las dos superpotencias discutieron bilateralmente sucesivos proyectos de tratado, hasta que convinieron en un texto unificado y lo sometieron formalmente a la consideración del Comité de Desarme. Allí aceptaron cambios "cosméticos", para emplear el término usual, pero en todo lo importante prevaleció su voluntad, como también prevaleció en la Asamblea General de las Naciones Unidas cuando en 1968 le dio su visto bueno, sin que tuviera la menor posibilidad de modificarlo. 3 .

El texto final del Tratado de No Proliferación de Armas Nucleares dejó mucho que desear, lo que fue explicable dadas las dificultades propias de la materia, los distintos intereses en juego y, sobre todo, la novedosa índole del objetivo del tratado, o sea vedar totalmente el desarrollo de un arma que algunos Estados ya poseían, cuando lo normal en todos los acuerdos de desarme anteriores y posteriores al TNP es que limiten o prohiban ciertas armas o técnicas a todos los Estados Partes en igualdad de condiciones.

No sólo el texto del TNP fue insatisfactorio sino que se desconocía por completo qué éxito tendría. De nada hubiera valido que en definitiva algunos lo ratificaran y otros no, ya que entonces estos últimos quedarían con todas las ventajas sobre los primeros, y el propósito mismo del Tratado quedaría desvirtuado. Habría mayor inestabilidad en las relaciones internacionales y la seguridad de todos se vería disminuida en vez de incrementada.

Era obvia la necesidad, transcurrido un plazo prudencial, de reexaminar la situación. Con ese propósito, el Artículo X, párrafo segundo, del TNP estableció especificamente que "Veinticinco años después de la entrada en vigor del Tratado se convocará a una Conferencia para decidir si el Tratado permanecerá en vigor indefinidamente o si se prorrogará por uno o más periodos suplementarios de duración determinada. Esta decisión será adoptada por la mayoría de las Partes en el Tratado".

El Tratado de No Proliferación entró en vigencia el 5 de marzo de 1970, o sea que la Conferencia prevista tendría que realizarse en 1995.

\footnotetext{
${ }^{3}$ El proceso estả ampliamente descrito en el libro El desarme de los desarmados - Argentina y el Tratado de No Proliferación de Armas Nucleares, (Buenos Aires: Editorial Pleamar: 1987), del autor de este articulo.
} 


\section{Las opciones.}

Cabe destacar $a b$ initio que el único punto que la Conferencia de Extensión tenía que tratar y decidir fue la prolongación de la vigencia del TNP. Hubo un discusión académica previa acerca de si la Conferencia podia o no resolver no extender el INP, es decir, poner término a su existencia, pero como se trataba de una eventualidad inimaginable; la misma no trascendió el plano de los planteos teóricos sin consecuencias. También se especuló acerca de qué ocurriria si en la Conferencia los Estados Partes, sin decidir abrogar el TNP, se revelaban incapaces de llegar a una decisión en un sentido o en otro, ${ }^{4}$ pero tampoco en este caso el tema fue debatido formalmente.

Hubiera sido lógico esperar que la Conferencia de Extensión estuviera capacitada para introducir enmiendas al TNP, particularmente porque la duración de su prolongación podria depender de que alguna $o$ algunas de sus cláusulas, fuente de repetidas objeciones, fuesen modificadas.

Las cosas en el TNP, sin embargo, nunca son normales. La Conferencia de Extensión no estaba habilitada para aprobar enmiendas, aunque -n teoria- un cuestionable manejo procesal podía hacer que la Conferencia se convirtiera en una Conferencia de Enmiendas de las previstas en el Artículo VIII, párrafo uno, del Tratado.

Pero aun esa eventualidad no habría tenido futuro. A diferencia de los otros tratados de desarme, el TNP es prácticamente inmodificable, como está universalmente reconocido. Los requisitos que el propio Tratado establece para que sea aprobada cualquier enmienda y luego para que la misma entre en vigor son tales que aseguran que, de hecho, ninguna tendrá éxito.

No deja de ser curioso que los autores del TNP hicieran prácticamente imposible enmendarlo. Ello podría explicarse en un primer momento, cuando ciertamente hubiera sido desaconsejable que sus disposiciones, que tanto trabajo costó redactar y negociar, pudiesen ser alteradas con relativa facilidad, máxime en circunstancias en que el futuro del propio Tratado era dudoso.

El transcurrir del tiempo evidenció, por una parte, que el éxito del TNP superó las más optimistas esperanzas de sus promotores, convirtiéndose en un instrumento internacional de especial solidez. A la vez, se fue

\footnotetext{
${ }^{4}$ William Epstein y Paul Szasz, "Extension of the Nuclear Non-Proliferation Treaty: A means of strenghtening the Treaty", Virginia Journal of International Law, Volume 13, Number 4, p. 754.
} 
haciendo cada vez más evidente que el articulado del Tratado daba lugar a numerosas críticas, en su mayor parte justificadas. El texto contenía reglas ambiguas, ofrecía vacíos y escapatorias (loopholes) y algunas de sus cláusulas habían sido ignoradas o mal aplicadas, sin hablar de su carácter inherentemente discriminatorio.

Una convención sólida pero asimismo duramente cuestionada en algunos aspectos estaba madura -ra lógico pensarlo-para ser reformada para satisfacción de todos, en primer lugar de sus numerosos Estados Partes. La posibilidad de enmiendas es, por otra parte, normal en cualquier tratado internacional. La Conferencia de Extensión podría haber facilitado ese proceso, si no adoptando directamente una enmienda, al menos disminuyendo alguna de las condiciones que lo hacen tan complicado. 5

Ocioso es señalar que la posibilidad de enmendar el Tratado jamás fue abordada en la Conferencia de Extensión. No solamente esa alternativa merece la oposición total de las potencias nucleares, con Estados Unidos a la cabeza, sino que tampoco estaba prevista expresamente en el Artículo X, segundo párrafo, del Tratado. En realidad, buena parte del debate que tuvo lugar durante los años previos a la Conferencia giró acerca de qué era lo que ésta podía hacer, en otras palabras, cuáles eran las opciones de que dispondrían los Estados Partes en 1995.

La discusión no fue sencilla, debido a que -como se ha dicho-6 "el Artículo $X$, segundo párrafo, es único, no teniendo equivalente en ningún otro tratado. Dado que su redacción es poco clara, resultado de una transacción alcanzada en negociaciones privadas realizadas durante un período prolongado, y dado también que las actas del Comité de Desarme de las Dieciocho Naciones no aclaran cuál es su verdadero significado, se requiere una amplia interpretación de su texto".

No entra en el campo de este trabajo analizar en detalle las diversas posiciones sostenidas en esta materia. Baste señalar que Estados Unidos

\footnotetext{
${ }^{5}$ En la $44^{*}$ Conferencia Pugwash sobre Ciencia y Asuntos Mundiales (Kolymbari, Creta, Grecia, 30 de junio-6 de julio de 1994), el autor propuso que se recomendara la introducción de una enmienda al TNP que eliminara un curioso requisito, el de que para que una enmienda entre en vigor es necesaria la ratifícación por parte de todos los Estados Partes miembros de la Junta de Gobernadores del Organismo Intermacional de Energía Atómica. Si bien ello no fue aceptado en su integridad, la Comisión I sugirió que en vez de la unanimidad de los integrantes de la Junta, se necesitase solamente una simple mayoria. Ese cambio hubiera hecho más factibje incorporar enmiendas al TNP, sin dejar de reconocer que se trató de un simple ejercicio académico, ya que era imposible esperar que la posibilidad de modificar el TNP fuese siquiera discutida. Pugwash Newsletter, Vol. 32, $\mathrm{N}^{\circ} 1$, julio 1994, p. 65.

${ }^{6}$ Epstein y Szasz, op. cit., p. 753.
} 
fue inflexible en afirmar que el referido Articulo $\mathrm{X}_{;} 2$, ofrece literalmente sólo tres opciones: extender el TNP indefinidamente, proriogarlo por un periodo de duración determinada o extenderlo por varios periodos sucesivos también de duración determinada. Como en los dos últimos casos, al término de la duración del período o periodos de prórroga, el TNP caducaría, lo que obviamente nadie quería, surge lógica la conclusión de que en realidad la única altemativa era la de extenderlo indefinidamente.

Frente a la posición mencionada precedentementé, clara a la vez que rígida, existió otra, con diversas variantes pero coincidente en un solo aspecto: la negativa a hacer del TNP un tratado permanente. En general, se propugnó condicionar su prórroga al logro de varias mediảas de desarme que la gran mayoría de la comunidad internacional viene reclamando desde hace largo tiempo: conclusión de un tratado de prohibición total de pruebas nucleares, otro sobre garantías negativas y positivas de seguridad, cese de la producción de material fisionable y destrucción del existente en la actualidad, amplia transferencia de tecnología nuclear, etc., $y$, principalmente, la realización de progresos substanciales y concretos en el campo del desarme nuclear, conducente a la eliminación final de este tipo de armas.

Las condiciones que se impondrían, expresa o implícitamente, a la extensión del TNP conllevaban obviamente su renovación por un periodo limitado, que permitiera controlar a su término si los pasos previstos se habian o no cumplido. La duración de esa prórroga varió en las diferentes propuestas, comenzando por lapsos de apenas un año o dos, pero en última instancia prevaleció la idea de extender la vigencia del TNP por otro período de veinticinco años.

Lógicamente, ello dejaba subsistente el problema de qué hacer cuando finalizara ese término de veinticinco años. Se podría -sostenia una fórmula presentada por Venezuela- efectuar una segunda Conferencia de Extensión y así sucesivamente. Se podría renovar automáticamente el TNP salvo que una mayoría de Estados Partes se pronunciara èn sentido negativo. Se podría suspender la Conferencia de Extensión y reanudarla posteriormente cuando se alcanzaran por lo menos algunas de las medidas arriba mencionadas, permaneciendo mientras tanto en vigor el TNP durante ese lapso que podía no ser breve.

Todas estas opciones fueron desechadas por Estados Unidos y sus partidarios, argumentando que no estaban previstas en el texto del ya citado Artículo $X$, segundo párrafo, y que, por lo tanto, adoptar alguna de esas fórmulas implicaba de hecho una enmienda del Tratado. 
Reseñadas ambas posiciones, cabe destacar dos circunstancias. Nadie, jamás, puso en duda la continuada existencia del TNP. Las divergencias se produjeron solamente acerca de si su continuación debía ser permanente $O$ a través de prórrogas sucesivas. En segundo lugar, la mención reiterada a Estados Unidos como líder de una posición no significa que fuera el único país que la defendía. Hubo quienes 10 acompañaron, sobre todo a partir de la intensa campaña realizada por el mismo, pero en todo momento fue evidente que el motor fundamental de esa postura quien la sostenía en toda oportunidad y en todo foro apropiado, de lejos el más vocal y firme partidario de la prolongación indefinida e incondicionada del TNP, fue siempre Estados Unidos.

\section{Las posiciones en disputa.}

Pocas conferencias como la de Extensión del TNP han estado precedidas de tan prolongado y variado debate. Lo que también es notable es que esas discusiones tuvieron lugar predominantemente en el ámbito académico no gubernamental. Infinidad de artículos en las revistas especializadas, multitud de discursos e intervenciones en foros competentes, conferencias y seminarios, precedieron durante años a la realización de la Conferencia de 1995. Salvo el caso de Estados Unidos, cuyos portavoces gubernamentales llevaron a cabo una verdadera cruzada en favor de la prolongación indefinida e incondicionada del $\mathrm{TNP}^{7}$ en general los demás países o no asumieron públicamente una posición hasta las vísperas de la Conferencia de Extensión ${ }^{8} \mathrm{O}$, si lo hicieron, no se dedicaron a publicitarla y fundamentarla en las numerosas reuniones que se ocuparon del tema.

De allí que el resumen que se hará a continuación de los principales argumentos esgrimidos para defender una y otra posición está basado principalmente en el debate esencialmente académico que tuvo lugar

\footnotetext{
${ }^{7}$ Funcionarios oficiales a todos los niveles bregaron insistentemente por la próroga del TNP, estando la conducción general de la camparia a cargo del Embajador Thomas Graham, de larga trayectoria en el campo del desarme. Fue Asesor Legal durante más de una década y también Director interino de la Agencia para Control de Armamentos y Desarme. En 1993, fue nombrado Representante Especial del Presidente de Estados Unidos con la tarea específica de ocuparse de la renovación del TNP.

${ }^{8}$ Argentina fue uno de los pocos paises que lo hicieron, al incluir el Poder Ejecutivo en el Mensaje que dirigió al Congreso el 7 de julio de 1994 solicitandó la aprobación del TNP, un párrafo en el que se expresó que la Argentina concurriria a la Conferencia de Extensión del TNP para, como objetivo primordial, "abogar por su prolongación indefinida".
} 
sobre esta materia, en revistas y ámbitos especializados. No cabe duda, sin embargo, que esas posturas reflejaban más que adecuadamente puntos de vista albergados en los departamentos competentes de cada gobierno, impresión reforzada por la participación, a veces, de funcionarioś oficiales que advertían no obstante que su presencia tenían carácter personal y no gubemamental.

Como se dijo anteriormente, la reiterada prédica de los representantes norteamericanos los convirtió de hecho en los máximos exponentes de la posición decididamente favorable a la prolongación sine die e incondicional del TNP. 9

Si uno analiza en detalle los argumentos expuestos en favor de la renovación indefinida del TNP arriba a la conclusión de que su fuerza convincente no llega a ser decisiva. Es cierto que, normalmente, todos los acuerdos de desarme son de duración permanente y era lógico entonces preguntarse por qué el Tratado de No Proliferación, superado exitosamente su período de prueba, no podía seguir igual camino que otras convenciones en el mismo campo. Pero también habría que reconocer que el TNP es un acuerdo válidamente objetable, en muchos. sentidos insatisfactorio a la vez que inmodificable, por lo que no desear su extensión sine die tenía razonable justificación.

Se sostuvo también que las potencias nucleares, y en particular Estados Unidos, habían cumplido acabadamente con los compromisos de desarme nuclear contenidos en el TNP, lo que es cuestionado por numerosos países que entienden que ese cumplimiento es insatisfactorio y que hay diversos pasos trascendentales que todavía están pendientes. ${ }^{10}$

A la crítica de que el TNP es discriminatorio, ya que divide al mundo entre quienes tienen el arma nuclear y quienes no la tienen, estableciendo derechos para unos y restricciones para los otros, los defensores de la posición à la que nos venimos refiriendo contestan que en realidad el Tratado no es discriminatorio, puesto que no inventó nada sino que se limitó a reconocer una situación preexistente, la posesión del arma nuclear en manos de cinco Estados. El contraargumento es que, de todos modos, el TNP legitima esa posesión y concede legalmente a esas cinco potencias un status privilegiado.

\footnotetext{
${ }^{9}$ Ver, por ejemplo, Thomas Graham, "The United States and the Prospects for NPT Extension", Arms Control Today, Volume 25, Number 1, Washington, January/February 1995, pp. 3-6.

${ }^{10}$ Las cosas son tan asi que, de las cinco Conferencias quinquenales de Revisión del funcionamiento del TNP, tres fracasaron por falta de acuerdo, inclusive la de 1995 que se celebró simultáneamente con la Conferencia de Extensión.
} 
Esta diferencia de puntos de vista se viene expresando casi desde el momento mismo en que el TNP entró en vigencia, y la Conferenciã de Extensión dio lugar a que la discusión se renovara. Es lógicamente materia opinable la medida en que cada argumento abona o no la conveniencia de prorrogar indefinidamente el TNP.

Es de destacar, sin embargo, que el núcleo de toda expresión de la primera posición, la favorable a la renovación sine die del TNP, se concentró en una exaltación de las virtudes del Tratado, reales y presuntas: que brinda seguridad para todos, que ha impedido la aparición de nuevos países con armas nucleares, que es el único instrumento que contiene un compromiso de desarmarse de parte de las potencias nucleares, etc.

Que el TNP tiene aspectos positivos, sería absurdo ignorarlo y, en general, todo el mundo lo reconoce. El problema son sus defectos y la imposibilidad legal de corregirlos. Pero -como hemos señalado anteriormente-nadie buscaba la desaparición del TNP, por lo que, en realidad, ensalzar las ventajas del mismo era absolutamente innecesario y el hecho de hacerlo sin motivo para avanzar la causa de su prolongación indefinida, combatiendo un enemigo imaginario, parece demostrar, en el fondo, la carencia de otros argumentos más sólidos y pertinentes.

Una reflexión objetiva conduciría probablemente a la conclusión de que, en última instancia, los efectos de una prolongación permanente del TNP o por un cuarto de siglo serían similares o que, en todo caso, aun admitiendo que para algunos la primera alternativa ofrece apreciables ventajas, las diferencias no son de tal magnitud como para explicar la casi obsesión con que Estados Unidos la promovió a ultranza. Su perenne argumento de que una prórroga no permanente del TNP lo debilitaria de tal manera que perdería fuerza y produciria un aumento de la inseguridad general no resiste un análisis serio, ya que si, con una supuesta "precariedad" de 25 años, el TNP se consolidó extraordinariamente y alcanzó el insólito número de 178 ratificaciones (de lejos la mayor cantidad de cualquier acuerdo de desarme), no se ve cómo, partiendo de una situación mucho más firme, otro período de 25 años tendría el efecto contrario. Parece razonable explorar si no hay detrás de esa actitud otras razones que las explicitadas públicamente.

Para comenzar, resulta indudable que, tal como fue redactado en 1968, el Tratado de No Proliferación satisface plenamente los intereses de Estados Unidos y, en general, los de las demás potencias nucleares. Les reconoce una situación monopólica, solamente tienen derechos y 
apenas dos obligaciones: una, la de no transferir a terceros armas nucleares o los conocimientos necesarios para producirlas, política que de por sí ya habían adoptado; y la segunda, un vago compromiso de realizar de buena fe negociaciones tendientes al desarme nuclear y al logro de la meta fijada ya en los albores mismos de la existencia de las Naciones Unidas: un tratado de desarme general y completo bajo estricto y eficaz control internacional (Artículo VI del TNP). Ese compromiso de desarme, por lo demás, no constituye una obligación específica de làs potencias nucleares sino que se extiende a todos los Estados Partes en el TiNP,' o sea prácticamente a la casi totalidad de la comunidad internacional.

El grueso de las obligaciones contenidas en el TNP se aplica a los Estados no nucleares, que ven vedadas sus posibilidades de adquirir armas nucleares y que son sometidos a ese efecto a un riguroso sistema de control internacional. Sería absurdo negar los beneficios que derivan, para la seguridad común y la individual de las naciones Partes, de la imposibilidad de que aparezcan en poder de nuevos países esas nefastas armas de destrucción en masa; pero también es innegable que los intereses de las potencias nucleares se ven particularmente favorecidos no sólo con el mejoramiento de la seguridad global sino también con la imposibilidad de que surjan nuevas potencias nucleares, que serían aun en el mejor de los casos innegables competidoras en la escena internacional. No se debe olvidar que la principal preocupación que se tuvo en cuenta al negociarse el TNP fue el peligro de que ciertos países desarrollados, en primer lugar Alemania y Japón, produjeran armas nucleares. Ese riesgo quedó definitivamente despejado al concluirse el TNP, como también quedó cerrado -salvo obviamente a través de violaciones al Tratado-el camino para que países de menores capacidades, como Corea del Norte, Irak, Irán y Libia, ingresaran también al pequeño círculo de poseedores del arma nuclear.

No cabe duda de que Estados Unidos está ampliamente conforme con el Tratado de No Proliferación tal como está. No es de extrañar que, como principal negociador del INP (conjuntamente con la otra superpotencia de entonces, la Unión Soviética), se haya asegurado de que el Tratado sea tan dificil de enmendar como para hacerlo, de hecho, inmodificable. 


\section{La prórroga indefinida.}

En 1968 las circunstancias determinaron que el TNP tuviera una duración limitada, veinticinco años. En 1995 llegó la oportunidad de convertirlo en permanente y de esa manera consolidar definitivamente el status de un instrumento internacional tan favorable a sus intereses. La posibilidad de que la prórroga del TNP fuera limitada, aunque el plazo fuera considerable, despertaba naturalmente tajante rechazo en primer lugar en las potencias nucleares. Ninguna quería tener que enfrentar nuevamente, dentro de cierto tiempo, la preocupación y el desgaste, sin mencionar el riesgo, que traería aparejados una segunda -y quizá luego una tercera y una cuarta-negociación sobre el futuro del TNP. Esforzarse al máximo para que el TNP no estuviera sujeto nunca más a un relativo cuestionamiento resulta entonces úna decisión muy fácil de comprender.

\section{La prórroga incondicionada.}

En líneas generales, en el amplio debate que sobre este tema tuvo lugar en los últimos años siempre se hizo hincapié en la prolongación indefinida del TNP, es decir, en su vigencia de carácter permanente, sin limitación en el tiempo. Sin desconocer la importancia de este punto, se ha dado menor relevancia a otro aspecto del problema, que tiene que ver con la prórroga incondicionada del TNP. Y ello ha sido un error, ya que esta materia estuvo en la esencia de la preocupación de Estados Unidos y sus aliados.

Uno puede pensar que las potencias nucleares hubieran podido en última instancia vivir con una prórroga limitada del TNP, sobre todo si era prolongada. Lo que era inaceptable era una continuación del TNP sujeta a condiciones, es decir, ligada a la conclusión de ciertas medidas concretas de desarme nuclear que un número no pequeño de países no nucleares entiende son indispensables en el marco del Artículo VI del TNP, al que anteriormente nos hemos referido. Aun cuando el logro de esos pasos no estuviera especificamente incluido en el texto de un acuerdo como requisito sine qua non para la continuada vigencia del TNP, hubiera quedado sobreentendido en toda extensión restringida del TNP que una nueva prolongación dependería en gran medida del juicio de la mayoría de los participantes en la futura conferencia acerca del grado en que las diferentes acciones propugnadas hubieran sido cumplidas. 
En todas las Conferencias de Revisión del funcionamiento del TNP este tema estuvo en el centro de los debates y divergencias ocurridos. Las posiciones se dividieron siempre entre aquéllos, con los países nucleares en el frente, que sostenían que los progresos logrados en esta materia eran satisfactorios, y quienes entendían lo contrario. La vaguedad de la formulación del Artículo VI del TNP da pie para defender puntos de vista diametralmente opuestos, pero la situación sería distinta si existiese un compromiso explícito o implícito que identificara inequívocamente qué pasos en el campo del desarme nuclear debían ser considerados como vinculados directamente a la operación del ya recordado Artículo. VI.

Un grupo numeroso de Estados no nucleares, englobados generalmente bajo la denominación de no alineados aunque en ciertos casos cabría discutir la propiedad de ese nombre, viene sosteniendo desde hace largos años la necesidad de llegar a tratados sobre ciertos temas: la conclusión de un acuerdo sobre la prohibición completa de ensayos nucleares, mencionado específicamente ya en 1968 en uno de los párrafos del Preámbulo del TNP; el otorgamiento a través de una convención de las llamadas garantías de seguridades negativas y positivas, que consisten, respectivamente, en que las potencias nucleares nunca amenazarán con armas atómicas a los países que han renunciado a ellas a través del TNP, y en que acudirán en auxilio de los mismos cuando se vean agredidos o amenazados con armas de ese tipo ; un acuerdo sobre cese de la producción de material fisionable apto para su utilización en armas nucleares y sobre la destrucción del ya existente; la eliminación de políticas restrictivas de las transferencias de equipos y de tecnología nucleares; declaraciones de todas las potencias nucleares sobre no primer uso del arma nuclear, etc.

Es quizá comprensible que numerosos países pretendieran obtener la realización de esos objetivos utilizando la continuación del TNP como elemento coactivo, pero también es comprensible la posición de quienes se opusieron a esa suerte de marchandage, señalando que el futuro del Tratado debía ser decidido de acuerdo con sus propios méritos y que debía ser juzgado sobre la base de su valor para la preservación de la seguridad internacional y como instrumento que obra en beneficio de todos y no exclusiva o primordialmente de las grandes potencias.

Sea cual fuera la verdad, dos realidades son incontestables: primero, que el peso de la negociación de los referidos acuerdos recae necesariamente en los países dueños de armas nucleares, aunque del proceso participen otros países; y segundo, que es explicable que tales Estados 
nucleares quieran decidir por sí mismos cuándo, cómo y de qué manera negociarán al respecto, sin verse constreñidos y menos obligados a hacerlo en virtud de condicionamientos impuestos para la renovación del TNP

En todo caso, resulta evidente que Estados Unidos no estaba de ningún modo dispuesto a aceptar alguna forma de obligación de negociar, para determinada fecha, acuerdo alguno relativo a los que juzga sus intereses vitales. No cabe duda de que las demás potencias nucleares opinaban lo mismo, pero en mayor o menor medida dejaron a Estados Unidos la responsabilidad de oponerse frontalmente a intentos de esa índole y de bregar por el contrario por la prolongación indefinida y sobre todo incondicionada del TNP. No es dificil de comprender en consecuencia la extrema importancia.que Estados Unidos otorgó a este tema y, al mismo tiempo, la dificultad en invocar públicamente sus intereses nacionales para defender su posición por lo que se vio obligado a utilizar argumentos más generales basados en las virtudes del TNP y, sobre todo, a elegir la presión diplomática como el medio más eficaz para alcanzar las metas apetecidas.

\section{La presión norteamericana.}

Como se dijo anteriormente, es normal $c_{\alpha}$ de en las conferencias internacionales, sobre todo las que tienen cierta importancia, los Estados ejèrzan; en la medida de sus posibilidades, alguna forma de presión para obtener un resultado favorable a sus intereses. Va de suyo que la eficacia de tales presiones dependerá en gran parte del poderío del país que las realiza, y en tal sentido es obvio que el de Estados Unidos tiene un peso muy dificil de ignorar. Pero lo que otorga particular relieve a la presión ejercida por Estados Unidos en relación con la Conferencia de Extensión del INP es: a) la antigüedad en el tiempo con que fue practicada; b) la inusual jerarquía de los funcionarios que se ocuparon del tema; y c) el inusitado vigor y la insistencia con que la presión fue llevada a cabo.

El comienzo de la campaña norteamericana puede fijarse en 1990, cinco años antes de la Conferencia. Ya en esa época Estados Unidos buscó -y obtuvo-el apoyo de sus aliados de la OTAN para la extensión indefinida del TNP, y luego lo consiguió también del Grupo de Siete Países Industrializados (G-7) y de la Conferencia sobre Seguridad y Cooperación en Europa. En 1993, a dos años de la Conferencia, el 
esfuerzo se intensificó y adquirió una dimensión universal con la designación de un alto funcionario con dedicación exclusiva a este tema, quien, con su equipo de colaboradores, empezó a recorrer el mundo tratando, en cada capital, de convencer a los respectivos gobiemos de que apoyaran la posición norteamericana. ${ }^{11}$

Hacia mediados de 1994, los funcionarios de nivel más alto en el gobierno de Estados Unidos empézaron a tomar participación directa en el esfuerzo para lograr la extensión definitiva del TNP. Lideraron la gestión el propio Presidente de la Nación, el Vicepresidente y el Secretario de Estado y ellos, unidos a los estratos superiores del Departamento de Estado y a los Embajadores distribuidos por el mundo; procuraron influir en favor del punto de vista norteamericano. Al hacer eso -se ha dicho- "la extensión indefinida del INP dejó de ser exclusivamente un tema de no proliferación y control de armamentos y se volvió -para Estados Unidos y sus interlocutores- una cuestión de alta politica, que debía ser tratada como tal" (énfasis del autor). ${ }^{12}$

En otras palabras, "en el momento en que el Presidente de Estados Unidos expresó en público que él encabezaría la marcha por la extensión indefinida (del TNP), un número mucho mayor dentro de la búrocràcia norteamericana recibió sus órdenes de avance. Ello convirtió lo que era una campaña tranquila de Mr. Graham en lo que llamaría una cruzada" (énfasis del autor). 13

La previsión de Estados Unidos al iniciar su campaña con tanta anticipación y al dar a la misma una inusitada energía dio sus frutos, quizá los más valiosos, cuando el Movimiento de Países No Alineados, reunido nada menos que en Bandung, ciudad tan intimamente asociada a su nacimiento, fue incapaz de adoptar una posición unificada con respecto a la prórroga del TNP. En circunstancias normales, la posición de la gran mayoría de los países no alineados en materia de no proliferación y desarme es parecida, si no idéntica, y hubiera debido favorecer la

\footnotetext{
${ }^{11}$ Dunn, Lewis, op. cit., p. 3. El trabajo de Dunn, que ha sido citado varias veces cn este artículo, resulta particularmente valioso e ilustrativo, porque está escrito por quien fue Director Asistente de la Agencia para Control de Armamentos y Desarme de Estados Unidos, estuvo conectado desde hace una década con la marcha del Tratado de No Proliferación, fue Asesor de la Delegación norteamericana en la Conferencia de Extensión de 1995 y, sobre todo, porque su propósito en este articulo fue describir objetivamente los preparativos y el desarrollo de la Conferencia y no, por cierto, formular críticas a la actuación de su pais.

${ }^{12}$ Dunn, Lewis, op. cit., p. 5.

${ }^{13}$ Deciaración del Embajador mexicano Miguel Marin Bosch. En Welsh, Susan, "Delegate perspectives on the 1995 Review and Extension Conference", The Nonproliferation Review, Volume 2, Number 3, Monterey Institute of Iniernational Studies, Monterey, Spring-Summer 1995, p. 7.
} 
imposición de diferentes medidas de desarme nuclear como requisito para la prolongación del TNP. El hecho de que ello no ocurrió en Bandung se debe, al menos en parte, a que un no pequeño número de países no alineados ya estaba "convencido" de la bondad de la prórroga permanente del TNP. La consecuencia fue "que el fracaso en producir un frente unido poco menos que garantizó la extensión indefinida del TNP", ${ }^{14} \mathrm{O}$, en otras palabras, "la falta de consenso en la conferencia de Bandung significa que la oposición del Movimiento de Países No Alineados a la extensión permanente del TNP se ha virtualmente desintegrado". 15

La situación fue descrita claramente cuando se dijo que "el resultado de Bandung fue un preludio de lo que iba a pasar aquí (la Conferencia de 1995), porque en Bandung Estados soberanos que estuvieron sujetos a presión obstrayeron un consenso en favor de la extensión sucesiva cađa 25 añosi Si ello ocurrió en un foro de no alineados, podía preverse lo que iba a pasar aquí". 16

La Conferencia de Extensión del Tratado de No Proliferación se inició en Nueva York el 17 de abril de 1995, con la participación de 175 de los 178 Estados Partes.

El hecho de que la Conferencia se celebrara en Nueva York y no en Ginebra, donde normalmente se reúnen los órganos de desarme y donde habían tenido lugar tanto la negociación del INP como sus cuatro Conferencias quinquenales de Examen de su funcionamiento $(1975,1980$, 1985 y 1990), merece en este contexto un comentario. Estados Unidos fue el principal interesado en ese cambio de sede. Cabe preguntar por qué. La explicación se halla en la realidad de que los pequeños paŕses; blancos prioritarios de la labor de convencimiento que la superpotencia ya había ejecutado o que proyectaba llevar a cabo en la Conferencia misma, generalmente no tienen, por su extrema carencia de recursos, Misiones Permanentes en Ginebra. Sí las tienen, en cambio, en Nueva York, puesto que su ingreso y posterior participación en las Naciones Unidas es, para no pocos de ellos, una de sus escasas muestras de presencia internacional. Si la Conferencia se hacía en Ginebra era probable que parte de ellos no tomara parte en la misma y, si esto era asi, Estados Unidos se vería privado de unos cuantos votos que hubieran apoyado su posición.

\footnotetext{
${ }^{14}$ Zamora Collina, Tom, "South Africa bridges the gap", The Bulletin of the Atomic Scientists", Vol. $51, N^{\circ} 4$, Chicago, July/August 1995 , p. 31.

${ }^{15}$ Medeiros, Evan, "Indefinite NPT extension likely as Conference nears crucial vote", Arms Control Today, Volume 25, Number 4, Washington, May 1995, p. 21.

${ }^{16}$ Welsh, Susan, op. cit., p. 8. Declaraciones del Embajador Adolfo Taylhardat de Venezuela.
} 
La campaña en búsqueda de la prolongación indefinida del TNP no cesó sino todo lo contrario con el comienzo de la Conferencia. "La intensidad de esta actividad continuó durante las semanas siguientes en las discusiones en Comités, maniobras de corredor, conversaciones bilaterales en cuartos de hotel y tráfico de telegramas en un sentido y en otro. 17 :

Es una innegable realidad que esos diálogos en pasillos y habitaciones de hotel no quedan documentados y se hace por el contrario todo lo posible para que no queden rastros de esas maniobras no siempre gratas. Siendo así las cosas, es verdaderamente extraordinario que las presiones ejercidas durante la Conferencia hayan trascendido con tanta frecuencia y seriedad y que algunos delegados no hayan hesitado en decirlo públicamente.

En términos coloquiales, uno de los participantes en la Conferencia manifestó personalmente al autor de este artículo que los americanos no hicieron solamente "arm twisting", para usar la expresión habitual, sino que llegaron hasta el "leg breaking".

El Embajador Ryukichi Imai de Japón, que fue delégado a la Conferencia de Desarme de Ginebra y estuvo vinculado durante años a 'numerosas reuniones sobre esta temática, pudo afirmar que no pocos países recibieron gestiones diplomáticas "como las que efectuó Estados Unidos ante México, diciéndole que votara en favor de la propuesta norteamericana o de lo contrario no habria ayuda de Estados Unidos para sostener el peso mexicano". En el mismo artículo Imai cita palabras que habria pronunciado un representante de un Estado pequeño: "Estamos sometidos a tanta presión que los países menores como el mío no. pueden resistirse a otorgar un voto favorable" ${ }^{18}$

El ejemplo de México podrá ser cierto o no, pero parece significativa la prudencia con que el experimentado especialista de ese país, Embajador Miguel Marin Bosch, contestó una pregunta que se le formulara al respecto. En realidad, es también significativo que se considerara pertinente efectuarle dicha pregunta. Marín Bosch declaró que el negociador norteamericano Embajador Graham visitó México para hablar del tema y que "no estableció ... un vínculo entre el llamado paquete de

\footnotetext{
${ }^{17}$ Dunn, Lewis, op, cit., p. 6.

${ }^{18}$ Imai, Ryukichir, "Implications of the result of the NPT Review and Extension Conference", Plutonium, Council for Nuclear Fuel Cycle, $N^{0} 10$, Tokyo, Summer 1995, p. 13.
} 
ayuda a México y un apoyo a la prórroga indefinida del TNP, por lo que no hay tal cosa... por lo menos en lo que yo sepa" (énfasis del autor). ${ }^{19}$

El Embajador Adolfo Taylhardat de Venezuela fue menos "diplomático". Había sido un activo vocero de la llamada "propuesta venezolana", que consistía en que el TNP fuera renovado cada 25 años a través de una conferencia equivalente a la de 1995 . Es de suponer que esa fórmula contaba con el apoyo de su Cancillería, razón por la cual llamó la atención que en el curso de la Conferencia el Embajador Taylhardat, jefe de la delegación de su país, recibiera instrucciones de no sólo apoyar la extensión indefinida del TNP sino también de copatrocinar la correspondiente moción. Ello motivó la renuncia inmediata de Taylhardat y explica sus amargas declaraciones posteriores: "En mi opinión, la decísión de copatrocinar fue adoptada a causa de la presión... Para mí fue una cuestión de dignidad". Agregó que "existió demasiada presión ... aplïcada en todas direcciones. Mi impresión es que si hubiera habido la posibilidad de tomar posiciones con libertad durante la Conferencia, la prolongación indefinida no hubiese sido aprobada, ya que los países preferían una opción diferente". ${ }^{20}$

El Embajador Nugrobo Wisnumurti, Representante Permanente de Indonesia ante las Naciones Unidas, estimó apropiado declarar que "lo que yo juzgo inquietante es la manera en que se alcanzó una mayoría en favor de la extensión indefinida. Fue sencillamente por medio del uso de tácticas de presión contra los países pequeños". ${ }^{21}$

Aunque la calidad de la relación entre Irán y Estados Unidos induce naturalmente a tomar con reservas las palabras de un miembro de la Misión Permanente de Irán ante las Naciones Unidas, otras referencias coincidentes como las ya recordadas y el tono en general moderado de las declaraciones de ese funcionario permiten asignar cierta base fáctica a expresiones como: "Una cantidad de presiones ... promesas y a veces amenazas ... fueron ejercidas sobre países no alineados ... por ciertos Estados nucleares, en particular Estados Unidos y ciertos paises occidentales... Algunos diplomáticos dijeron muy explícitamente que presiones habian sido aplicadas sobre sus países. Por ello, esto permite cuestionar

\footnotetext{
${ }^{19}$ Welsh, Susan, op, cit., p. 7. La serie de entrevistas que condujo Susan Welsh al término de la Conferencia, en procura de recoger impresiones sobre su desarrollo, no tuvo como propósito particular recoger referencias al ejercicio de presiones durante la misma y evidencia de ello es que varios de los entrevistados no aludieron a ese tema. El hecho de que otros delegados consideraran apropiado destacar ese aspecto de la Conferencia es sin embargo sintomático.

${ }^{20}$ Ibid, , p. 8.

${ }^{21}$ Ibid., p. 6.
} 
el método por cuyo intermedio fue alcanzada la prórroga indefinida del

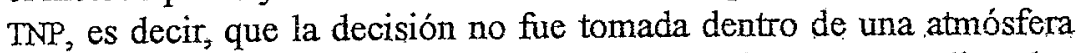
saludable, ni de manera democrática. Si los miembros no alineados hubiesen tenido la oportunidad de expresarse librenente acerca del problema de la extensión, estoy seguro de que hubieran actuado de otra manera" 22

Ejemplos como los que se termina de citar podrian multiplicarse. Por sí mismos no prueban la absoluta verdad de lo que afirman, pero al menos son síntema y evidencia de una percepción generalizada acerca de qué ocurrió durante la Conferencia de 1995 y en la época precedente. Incluso lo admitió Thérese Delpech, alta funcionaria del Comisariado de Energía Atómica de Francia, cuando, interrogada sobre el uso de presión en esta materia, contestó que "efectivamente, eso había sido dicho en diferentes ocasiones durante la Conferencia". Si bien manifestó no compartir esa aseveración, parece algo ingenuo el argumento dado para ello: "las Partes en el TNP son Estados soberanos", como si esa condición los hiciera inmunes a toda clase de presión externa. ${ }^{23}$

Todo lo que se termina de exponer no significa naturalmente que todos los países que se pronunciaron en favor de la extensión indefinida del TNP lo hicieron como resultado de la presión norteamericana. Algunos compartían esa posición, empezando por Sudáfrica, país que tuvo una actuación preponderante en la Conferencia ${ }^{24}$ al proponer que, como una suerte de compensación por la prórroga sine die del TNP, la Conferencia acordara un examen periódico más severo de su funcionamiento y una serie de Principios sobre No Proliferación y Desarme que debian ser observados en ese contexto. La fórmula sudafricana fue aceptada (aunque suavizada por exigencia de los países nucleares y sus aliados) y facilitó grandemente el resultado final de la Conferencia.

El ejemplo de Sudáfrica es demostrativo, porque, como dijo un miembro de su delegación a la Conferencia, "¿Qué se puede hacer cuando la posición que uno adopta sobre la base de sus intereses nacionales es también la posición que los Estados Unidos quiere que uno tome?". Las

\footnotetext{
${ }^{22}$ Ibid., p. 13. Declaración de Behrouz Moradi, funcionarịo de la Misión Permanente de Irạ́n ante. las Naciones Unidas.

${ }^{23}$ Ibid., p. 20.

${ }^{24}$ "Mucho del mérito por la disminución de las tensiones entre los paises nucleares y no nucleares en la conferencia de Nueva York se atribuye al Ministro de Relaciones Exteriores de Sudáfrica, Alfred Nzo, por presentar el 19 de abril una fórmula que podia tender un puente entre los abogados de la extensión indefinida del TNP y los paises que mantenian firmes y diferentes posiciones acerca de esa opción". En Medeiros, Evan, op. cit., p. 21.
} 
palabras del diplomático sudafricano son indicativas de la particular situación de su país en este punto, pero al mismo tiempo son señal de que las presiones existieron. "Fue de conocimiento público durante los meses anteriores a la Conferencia que los Estados Unidos estaban empleando una diplomacia silenciosa de "carrots or sticks" en relación con muchos países para consolidar el apoyo a una extensión indefinida". El Embajador de Estados Unidos en Sudáfrica habría destacado que una posición equivocada de ese país respecto del TNP "afectaría la opinión de Estados Unidos con respecto a las credenciales de Pretoria en materia de no proliferación" y "dificultaría la disposición de los Estados proveedores de asistencia nuclear para actuar en el área de la cooperación nuclear para fines pacíficos". 25

A esta altura, puede decirse que la existencia de presiones, quizá excesivas, antes y durante la Conferencia de Extensión del TNP está demostrada. Cabe agregar que, aunque el origen de las presiones estuvo radicado en los Estados Unidos, este país no fue el único que las ejerció. Algunos de sus aliados de la OTAN también las aplicaron en los países que juzgaron receptivos. Francia las efectuó indudablemente en los países africanos ex-colonias francesas, en los que su influencia -regún es de público conocimiento en los círculos de las Naciones Unidas-sigue siendo considerable. Rusia hizo lo mismo en las naciones que formaron parte de la ex-Unión Soviética.

Incluso países como Canadá desempeñaron un papel importante en la Conferencia de 1995. Quizá no ejerció presión pero sí se convirtió en el gestor de la posición favorable a la prórroga permanente. Como dijo Christopher Westpal, Embajador para Desarme de la Cancillería canadiense, "se solicitó a Canadá que tomara el liderazgo en promover la extensión indefinida" (énfasis del autor). No hace falta ser muy perspicaz pära imaginar quién solicitó a Canadá que asumiera ese papel, que dada su condición de país no poseedor de armas nucleares éstaba en inmejorảble situación para desempeñar El Embajador Westpal añadió que "yo personalmente trabajé a tiempo completo en el TNP durante seis meses... Parte de nuestros esfuerzos incluyó apuntar (target) sistemáticamente a 74 países indecisos para persuadirlos -uno a uno, a través de reuniones y almuerzos celebrados a invitación de nuestro Embajador ante las Naciones Unidas y a través también de repetidas gestiones realizadas por nuestros Embajadores en el exterior-de que, según el punto de vista de

${ }^{25}$ Zamora Collina, Tom, op. cit., p. 31. 
Canadá como país no dueño de amas nucieares, la extensión indefinida estaba convincemente justificada" 26

Podría agregarse otro elemento de juicio para abonar la innegable presencia de factores de poder en el desarrollo de la Conferencia. En las reuniones del Comité Preparatorio y en los prolegómenos de la misma Conferencia hubo una discusión acerca de si el voto para la prórroga del TNP debía ser público o secreto, es decir, por escrito. Lo normal es lo primero y así lo sostuvieron los delegados favorables a la prolongación permanente. Quienes tenían otra posición, preferian el voto escrito. Esa aparente discusión procesal tenía sin embargo un trasfondo. Los países que habían sido objeto de presión o que deseaban simplemente emitir un voto negativo temían las consecuencias de un voto público. ¿Por qué? Porque al ser público, su actitud quedaría fehacientemente registrada y los haría pasibles -según su apreciación-de las sanciones que presumiblemente les aplicarian las grandes potencias.

No se llegó a un voto, por dos razones: primero, la habilísima conducción del Presidente de la Conferencia, Jayantha Dhanapala, Embajador de Sri Lanka en los Estados Unidos ${ }_{2}^{27}$ que logró evitar que el voto fuera necesario; $y$, segundo, el progresivo deteriorò de la posición favorable a la prolongación limitada y condicionada del INP y el simultáneo fortalecimiento de los partidarios de su permanencia, lo que llevó a los primeros a aceptar la fórmula de Dhanapala, es decir, tomar nota de la existencia de una amplia mayoría en favor de la prolongación sine die del TNP y aprobar los ya mencionados Principios y reformas al proceso de revisión del funcionamiento del TNP, que, aunque compromisos políticos y no jurídicos, constituyeron una suerte de "consuelo" para la postura derrotada o, en la visión de algunos, la cubierta dulce que facilitó tragar una píldora amarga.

Es indudable que el resultado de la Conferencia significó una nítida victoria para Estados Unidos y para los que pensaban como ese país, sea por convicción, sea como consecuencia de presiones. Al mismo tiempo, parece también indudable que en el ánimo de numerosos países, quizá no una mayoría pero sí una cantidad respetable, quedó un mal gusto, una sensación de que las cosas no habían andado por cauces normales y de

\footnotetext{
${ }^{26}$ Welsh, Susan, op. cit., p. 4.

${ }^{27}$ Para disipar cualquier suspicacia, cabe señalar que el Embajador Dhanapala, especialista en desarme que fue Representante de Sri Lanka en la Conferencia de Desarme de Ginebra y Director del Instituto de las Naciones Unidas de Investigaciones sobre Desarme, habia sido seleccionado para presidir la Conferencia de 1995 desde mucho antes de su nombramiento como Embajador en Estados Unidos.
} 
que se llegó al resultado final de la Conferencia por vías no ortodoxas. Quizá la conclusión habría sido la misma de todos modos, pero sería faltar a la verdad si se dijera que todo el mundo quedó conforme con el desamollo de la Conferencia y con su saldo final.

En particular, quedó la impresión de que los países dueños de armas nucleares salieron de la Conferencia sin haber visto afectados sus intereses en lo más mínimo. Las concesiones "cosméticas" que se vieron obligados a otorgar no implicaron "un compromiso mayor que el que existía hace ya diez años en favor de una reducción planificada y por etapas de sus armas nucleares". 28

La frustración o la insatisfacción que, como se dijo, quedó en el espíritu de muchos participantes de la Conferencia, se vio fơrtalecida cuando, apenas cuatro días después de la conclusión de la Conferencia, China hizo estallar un bomba nuclear de ensayo. Semanas después, el Presidente de Francia anunció la realización por parte de su país de una serie de pruebas nucleares en el atolón de Muroroa. Lo impactante fue que esos hechos ocurrieran tan pronto después de una conferencia en la que los Estados nucleares habían hecho una serie de promesas, inclusive la de comportarse con la "máxime contención (utmost restraint)" en la materia. Tampoco fue alentador su compromiso de llegar a la eliminación total de las armas nucleares (meta que propugna enérgicamente la gran mayoría de los países no nucleares) como el objetivo final (ultimate goal) de todo el proceso de desarme nuclear. Objetivo final quiere decir en realidad -en la visión de muchos-para las calendas griegas o el siglo XXI o XXII o el que sea.

El propósito de este artículo no es en realidad formular juicios de valor sobre el resultado de la Conferencia de Extensión del Tratado de No Proliferación. La intención fue poner de manifiesto el ejercicio de una presión quizá exagerada en favor de la prórroga indefinida e incondicionada del TNP. Como se dijo, es muy dificil, dada la particular índole de las tratativas, aportar pruebas concluyentes en ese sentido. Las múltiples referencias efectuadas $-\mathrm{y}$ otras muchas que podrían haber sido agregadas- permiten apreciar, con buen fundamento, la existencia de tales presiones. Parece obvio asimismo que esas presiones tuvieron notable influencia en la adopción por parte de la Conferencia de una decisión en el sentido buscado.

\footnotetext{
${ }^{28}$ Welsh, Susan, op, cit, p. 8. Declaraciones del Embajador Marin Bosch de México, pais que terminó por no oponerse a la prolongación indefinida del TNP.
} 
Como se manifestó al comienzo de este artículo, el ejercicio de presiones en el curso de una conferencia es algo corriente pero criticable solamente en el mundo ideal de los principios. Sería hipócrita condenarlas y rasgarse las vestiduras por ello.

También es cierto que en no todos los foros internacionales se producen presiones, aun por parte de los países más poderosos. Al mismo tiempo, es asimismo innegable que en ciertos casos se va al otro extremo, $\mathrm{y}$ las presiones no solamente se ejercen sino que tienen una insistencia en el tiempo y un vigor que a veces parece desproporcionado. En mi opinión, esto último sucedió en relación con la Conferencia de Extensión del TNP.

La decisión de prorrogar indefinida e incondicionalmente dicho Tratado satisfizo a muchos, aunque probablemente a no todos los que se expresaron en favor de esa medida. Lo que parece cuestionable es la manera con que se arribó finalmente a esa solución. Sin poner las cosas en un plano de moralismo irrealista, parece indudable que el saldo dejó mucho que desear. Los intereses de las potencias nucleares y sus aliados se vieron ampliamente satisfechos. Cabe dudar de que lo mismo haya ocurrido con el resto de la comunidad intemacional. En muchos ámbitos quedó un sabor desagrạdable, cuya real magnitud y consecuencias prácticas es muy dificil apreciar. Los ensayos nucleares de China y Francia contribuyeron innegablemente a consolidar esos puntos de vista.

El tiempo dirá qué futuro tendrá la operación del TNP y las Conferencias de Examen de su funcionamiento. Como es imposible mejorar su texto y como ya está decidida su prolongación sin límite en el tiempo, podría pensarse entonces que poco o nada queda por hacer a su respecto; pero precisamente esa aparente imposibilidad jurídica de adoptar medidas correctivas puede en última instancia ser contraproducente y perjudicar de algún modo el porvenir del Tratado.

En opinión de este autor, el uso excesivo de presiones es responsable, en alguna medida, de ese sentimiento de inquietud y descontento que perduró en el ánimo de muchos participantes en la Conferencia de 1.995, lo que no deja de ser paradójico cuando la acción final adoptada por ella fue exactamente la misma que, aparentemente, propugnaba la amplia mayoría de los Estados Partes. Puede quizá presumirse, entonces, que su posición no fue todo lo auténtica que hubiera sido de desear. 